\title{
Factors affecting clinical referrals to the medical library
}

\author{
Liz Kellermeyer, MSLS \\ Biomedical Research Librarian \\ Library \& Knowledge Services \\ National Jewish Health \\ Denver, CO \\ Matthew J. Strand, PhD \\ Professor \\ Division Head, Biostatistics and Bioinformatics \\ National Jewish Health \\ Denver, CO
}

\section{ABSTRACT}

Objective: To determine why and how often clinical care teams refer patients to the medical library and to determine facilitators and barriers to referral.

Methods: A 2018 survey of clinical care teams at a research hospital measured awareness of library services available to patients, facilitators and barriers to referral, and likelihood of future referral. Spearman correlations were used to determine the strength of relationships between familiarity with the services and how often respondents referred those services. Referral rate distributions were compared between job type groups.

Results: Overall, self-reported referral rates were low. There was a marginally significant relationship between referral rate and job type $(p=0.01)$, with providers having lower referral rates. There was a positive correlation between familiarity with library services and service referral frequency ( $r_{s}=0.78$ for combined data) and between current referral rates and likelihood of future referral $\left(r_{s}=0.43\right.$ for combined data, $p<0.0001$ for both). Among respondents who had never referred patients, the top reasons were lack of awareness of library services and uncertainty about how to make referrals.

Conclusions: The results suggest that lack of knowledge, rather than lack of interest and support, results in lower clinic referrals to the library. Providers who are aware of the library services refer patients. Similarly, providers who currently refer are more likely to continue making referrals in the future. The qualitative responses show agreement, linking the lack of referrals to marketing and procedural insufficiencies, which indicates potential for increasing referrals by addressing these deficits. A streamlined patient referral system from clinic to library could be beneficial. 


\section{INTRODUCTION}

Library \& Knowledge Services in the Gerald Tucker Memorial Medical Library supports the clinical, research, and educational programs of National Jewish Health $(\mathrm{NJH})$, a nationally recognized respiratory hospital in Denver, Colorado. One goal of the medical library's mission is to provide health information resources for patients and their families. This goal reflects NJH's institutional mission of providing individualized care to patients and improving patient health outcomes by appropriately centering health care decisions around individual patient preferences and needs. NJH provides opportunities for promoting patient engagement and health literacy through several avenues, including providing online access to health records, publishing consumer health information on conditions NJH treats, and teaching courses on self-management of chronic disease.

The medical library is a unique resource for patients, offering librarian-mediated searches on specific health topics, guidance on finding authoritative health information, and help finding provider-recommended literature. While patients may find out about library services through a variety of sources, of particular interest are direct referrals from patients' clinical care teams. The clinical care team is defined here as physicians and clinical staff who have direct contact with patients and have opportunities to discuss patients' care with them. The clinical care teams are in a position of authority, and patients are likely to value their directives, which can positively impact patients' willingness to seek health information outside the office setting [1]. Physicians or other members of the care team may not always refer patients to the medical library to further investigate inquiries or curiosities, and the reasons for this are unclear. This study surveyed clinical care team members at NJH on their knowledge and referral frequency of medical library services oriented towards patients.

\section{LITERATURE REVIEW}

Evidence shows that patients who are engaged in their healthcare experience more satisfaction and have more positive health outcomes than those who are not [2]. One of the elements of this engagement is for patients to be adept at gathering information on their own and incorporating it into their lives. Higher health literacy rates have been associated with improved self-management of diseases and better medication compliance [3]. Hospital libraries that provide consumer health information and services are well-positioned to improve the health information literacy skills of patients [3]. As a service that provides both access to consumer health information and librarian-mediated searches, the medical library can attend to patients' individualized information needs. Librarians can supply specific physician-recommended articles, books, or handouts to a patient. Additionally, librarians have unique skills to lead patients through a reference interview, which often uncovers additional information needs. The medical library is not simply a dispensary of curated materials but a service 
that can instruct and empower patients to engage in efficient and effective informationseeking behaviors.

However, connecting patients to library services and resources is a challenge. In 2002, the National Library of Medicine introduced the Information Prescription (Information Rx) program as a possible facilitating practice. Under the Information Rx model, the physician prescribes specific reading material to patients, who are then expected to obtain and read the recommended material, sometimes with librarian mediation. These programs had a defined curriculum where physicians were trained and then given specific tools (e.g., information prescription pads) to be used when giving patients resource recommendations [4]. Among the positive outcomes associated with several pilot programs based on this model was the fact that physicians found value in library services and resources for their patients and thought they enhanced patient education and provider-patient communication [1, 3]. Some programs were able to successfully create a referral pathway that included the library $[5,6]$. While the outcomes of the programs showed that clinical care teams considered medical library resources valuable for patients, attempts to standardize a referral system from clinic to library have been complicated. For these early pilot programs, barriers to success included issues around physician adherence, patient participation, and the overall difficulty of sustaining collaborative efforts between several departments [7, 8]. Some newer iterations of the Information Rx model include the integration of the electronic health record (EHR) system to replace the outdated paper prescription pad [9, 10]. In these models, the clinical care team puts in specialized requests for information for their patients, which librarians fulfill. These programs cited lengthy technical hurdles to overcome, though both reported on ultimately positive outcomes, where bonds between departments were strengthened and the quality and volume of valuable health information to patients was increased $[9,10]$.

The literature does not discuss libraries and hospitals that lack a formal program or designated referral pathways for patients from physician to library. In such situations, it is unclear whether clinical care teams know about available library services or recommend them to their patients. The aim of this study was to understand why clinical care teams do or do not refer their patients to the medical library, the frequency of referral, and what barriers and facilitators are present in the process.

\section{METHODS}

A census of NJH clinical care teams was taken from October to December 2018. A survey was designed and administered online using REDCap and piloted by a small group of physicians and nurses. The final survey was sent to 482 employees identified as providers or clinical staff who have direct contact with patients and opportunities to discuss patients' care with them. An incentive was offered for each completed survey of a $\$ 5$ donation made to the Morgridge Academy, an on-campus K-8 school at NJH 
serving children with chronic illnesses. From the population, 95 responses were collected, representing a $19.7 \%$ response rate.

The survey featured multi-choice questions and Likert-type scales to measure awareness of library services available to patients, the facilitators and barriers to referral, and the likelihood of future referral. The focus was on five key services that the library provides for patients: patient portal account set up and questions, access to health information related to a diagnosis, librarian consultations or expert searching, physical library resources unrelated to health information (e.g., computers, printers), and archives or legacy information related to the hospital (e.g., annual reports). Numerical values were assigned to the frequency answers, ranging from 1 ("Never") to 6 ("Almost Every day"), and the mean scores were calculated for each service.

Demographic variables included gender, age, professional role, and years employed at the hospital. Spearman correlations were used to determine the strength of relationships between familiarity with the services and how often respondents referred those services $\left(r_{s}\right)$. Referral rate distributions were compared between job type groups using the Kruskal-Wallis test. This study was determined exempt by the IRB at NJH (HS-3203).

\section{FINDINGS}

Over half of the respondents (53\%) identified themselves as providers (physicians, physician's assistants, or nurse practitioners), with the next largest group being licensed nursing staff (19\%). Medical assistants, social workers, and an "other" category composed the remaining group (14\%). Fourteen percent did not answer this demographic question.

Overall, self-reported referral rates were low, with $57 \%$ of respondents saying that they have never referred a patient to the medical library, and only $14 \%$ saying they refer patients at least once a month. The highest referral rates were for "quick" services-those that don't require librarian expertise but are easy to remember and offer fast help to a patient. Other more specialized services, such as archives research or librarian-mediated expert searches, had the lowest referral scores. Across all services, the top two reasons clinical care teams referred patients to the library were that they felt the service adds value to the patient's visit and that the service complements or enhances the patient's access to health information.

Looking at the strength of relationships between familiarity with the services and service referral frequency, there was a positive correlation across all services $\left(r_{s}=0.78\right.$ for combined data, $p<0.0001$ ) (Figure 1). 


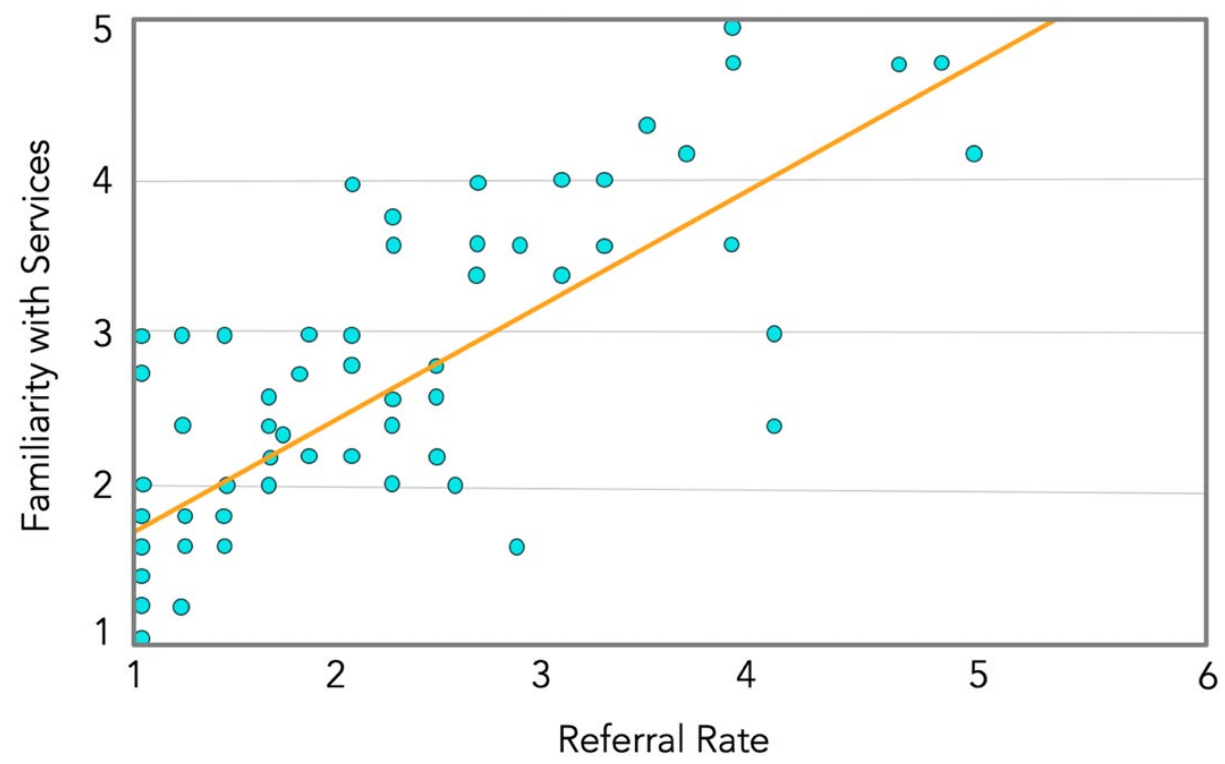

Figure 1. Relationship of clinical care team knowledge of library services and referral rate. (Familiarity scale: 1=Not Familiar, 2=Slightly familiar, 3=Somewhat familiar, 4=Moderately familiar, 5=Very familiar; Referral scale: 1=Never, 2=Less often than once a year, 3=A few times a year, 4=A few times a month, 5=At least once a week, 6=Almost every day)

The highest correlations occurred for librarian consults, health information, and patient portal help (Spearman correlations $=0.75$ or $0.76, p<0.0001$ ), and slightly lower correlations for archives and physical library space (Spearman correlations $=0.665$ and $0.60, p<0.0001)$. A positive correlation existed between current referral rates and likelihood of future referral $\left(r_{s}=0.43\right.$ for combined data, $\left.p<.0001\right)$. The more familiar respondents were with these services, the more often they were likely to refer them to patients. Additionally, those who referred in the past were more likely to say they would refer patients again. There was a marginally significant relationship between referral rate and job type $(p=0.01)$, with providers having lower referral rates (Figure 2$)$. 


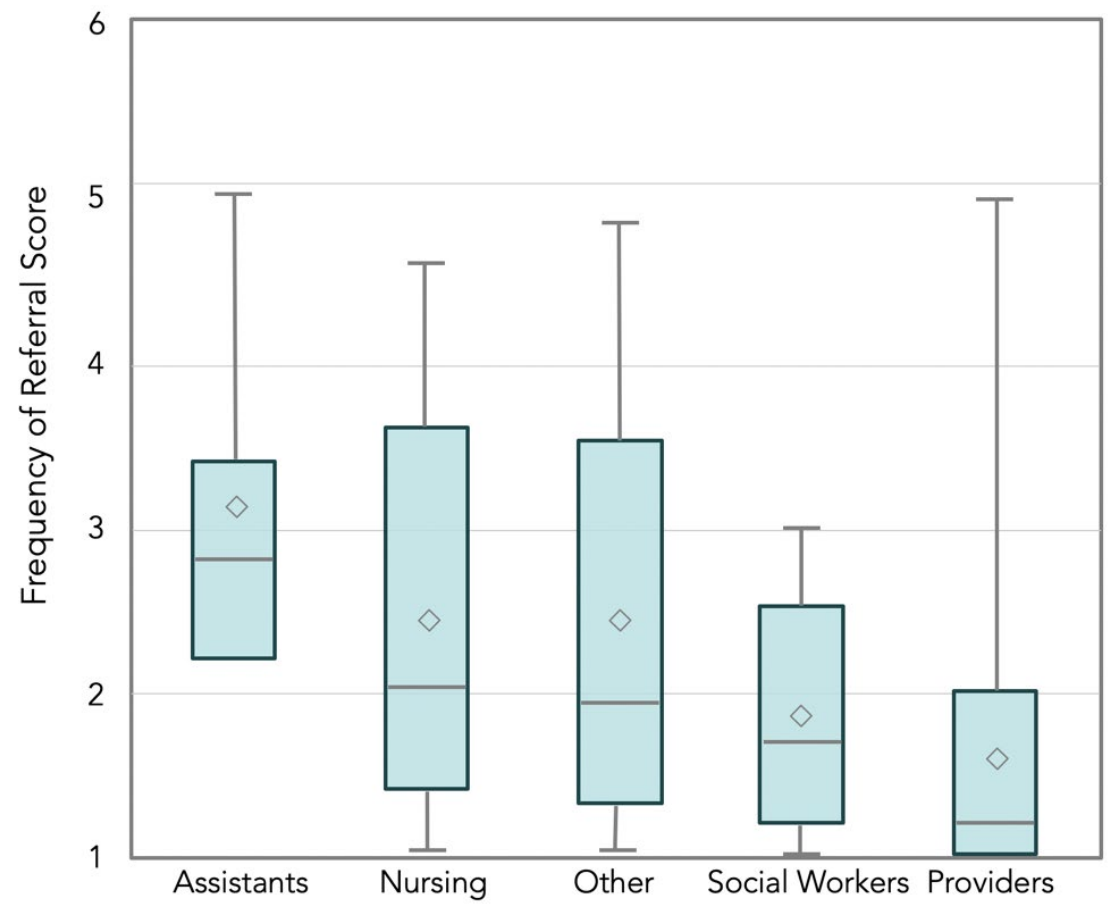

Figure 2. Relationship between referral rate and job type. (Referral scale: 1=Never, $2=L e s s$ often than once a year, 3=A few times a year, 4=A few times a month, 5=At least once a week, 6=Almost every day)

Among respondents who had never referred patients, the top reasons were "Not aware that library offered this service" (48\%) and "Don't know how to make a referral to library" (20\%); among the least selected reasons were "Clinic wants to control information to patient" $(2 \%)$ and "Concerned about quality of information provided by library" $(0 \%)$. Top referral reasons were "Complements or enhances the patient's access to health information" (28\%) and "Adds value to the patient's visit" $(27 \%)$. There was no significant correlation found between how often a person referred and how long they have been working at the hospital.

\section{DISCUSSION}

\section{Conclusions}

The results suggest that lack of knowledge, rather than lack of interest and support, results in lower clinic referrals to the library. The correlation between knowledge of services and likelihood of referral demonstrates that providers who are aware of library services do refer patients. Similarly, those currently referring are likely to make future referrals, suggesting these providers value library services. When asked why they refer patients, the care teams selected reasons that reflect their belief that the library has something of value to give to the patients. 
The qualitative responses show agreement, linking the lack of referrals to marketing and procedural insufficiencies (rather than distrust or dislike of the services), which indicates potential for increasing referrals by addressing these deficits. Respondents who had referred patients in the past demonstrated an understanding of what the library offers, citing librarian services and quiet space. One respondent stated, "I feel [visiting the library] is the best way for patients to do their research rather than online by themselves," which speaks to the value placed on the specialty of librarian services and on the willingness of clinic staff to look at the library as a collaborator on patient health.

Many of the comments from those who had not referred patients were variations of the fact that they were not aware of the services or do not think about them over the course of a visit. Some were not sure what differentiated library services from patient education already provided at a clinic visit. One respondent wrote, "Nursing provides [institutionally branded medical information sheets] and the team often prints and gives an article to the family if they have something they want to share." While there may be overlap between the kind of educational material patients receive from their care team and those a librarian may help them find, the difference between these two sources gets at the heart of encouraging health literacy. In the clinic setting, information is provided to the patient based on what the care team member wants the patient or family to know. In the library setting, the question always starts with what the patient wants to know. This idea of being able to articulate inquiries on behalf of one's own health and to take steps to obtain relevant resources are key to empowering patients to take an active role in their health.

\section{Marketing}

Inherent in the idea of marketing the library is the idea of communicating not just services but the library as a collaborative partner in improving health outcomes for patients. For this to be done, the clinic must understand what the library provides as distinct from what they provide, and providers must appreciate how the interaction may add value to the patient's visit. Because there is some evidence that clinical teams may be distrustful of librarians to provide accurate health information, the survey asked respondents about this [4]. However, in this survey $0 \%$ of the respondents selected this as a reason why they hadn't referred patients, so it is hopeful that the positive reputation of the medical library is good groundwork for growth.

Interestingly, no significant correlation was found between how often a person referred and how long they have been working at the hospital. While it was initially hypothesized that people who had worked at the hospital longer would retain knowledge about library services, this result suggests that marketing the library has done in the past has not necessarily been retained. More persistent communication with the clinic might be necessary in order to see sustained results. There may be some practical 
steps the library can take to bridge the gap between interest and awareness in services. It seems evident that focusing only on one marketing avenue is not sufficient and that spreading information about library services using varied tactics would increase the chance that care teams would be aware of the services. As a starting point, printed material could be distributed at new employee orientation and also placed in key work areas, such as clinic workstations. Librarians can also make a point to get on the agendas at key departmental meetings periodically, where they can remind faculty and staff about patient services available at the library. It can be advantageous to look for opportunities for integrated messaging across services. For instance, librarians are now invited to discussions about changes in patient portal processes, and in turn they are included in messaging from the information services department about where patients can seek portal setup help.

\section{Referral system}

For those who answered that they had never referred a patient to the medical library before, the second most frequent response (20\%) was that they were unclear about how to make such a referral. There is currently no formal referral system in place at the hospital; in order for a patient to get to the medical library via the clinical care team, the recommendation must come organically, based on the team member's knowledge of the library's services and a belief that the patient can be helped through a visit. If there is to be a collaboration across departments, a system is needed to support the workflows and tendencies already established in each area.

Outdated systems, such as the Information Rx program, rely on tools like prescription pads that may not even be in frequent use anymore. How can library services be streamlined into clinic workflow? One possibility is integrating medical library services into an EHR system, with the ability for providers to order librarian consults through the platform. Some such systems are already in place, with providers able to request a library collaboration by entering an order in the EHR. Librarians access these orders along with the medical record and can provide personalized information packets based on specific needs [9, 10]. An integrated system also presents the potential for charting and documenting library intervention, which could lead to the ability for larger data-driven studies looking at correlations between librarian interactions and patient outcomes. Libraries that have successfully set up such a system note the importance of securing a champion on the physician side of things, who can help guide the project and assure its goals and outcomes are aligned with those of the clinical care teams $[7,10]$.

\section{Limitations and future research}

Because this research was conducted only within one hospital setting, it is unknown whether these trends would be found widely among other medical libraries 
that serve a patient population. The survey instrument developed could be used elsewhere, and it may be beneficial to replicate the research in other settings to see if findings match those of this study. One limitation of this research was the exclusive focus on hospital staff rather than patients themselves. The results reveal only the intentions and behaviors of the clinical staff and not the patients' responses to referrals. Future research could look at patient populations using medical library services and examine their reasons for seeking out the resources, and trace whether their visits are motivated by clinical care team referrals. A combination of surveys would paint a more complete picture of the referral pipeline, measuring the difference between referral rate and compliance.

Those medical libraries that have referral systems incorporated into EHRs may be an obvious place to pick up the ideas in this research while working with electronic data. This research has shown there is interest in clinical care teams collaborating with the medical libraries to enhance patient care. The main barriers to referrals seem to be lack of knowledge of library services and an unclear referral pathway, which provides libraries and hospitals specific areas to focus on for improving referral rates.

\section{ACKNOWLEDGEMENTS}

Thank you to the MLA Research Training Institute for its training, support, and encouragement to carry out this research. This project was made possible in part by the Institute of Museum and Library Services (RE-95-17-0025-17).

\section{AUTHOR CONTRIBUTIONS}

L.K. designed and carried out the research, contributed to the analysis of results, and wrote the manuscript; M.S. processed the data, performed analysis, aided in interpreting the results, and contributed to writing the manuscript.

\section{REFERENCES}

1. Siegel ER, Logan RA, Harnsberger RL, Cravedi K, Krause JA, Lyon B, Hajarian K, Uhl J, Ruffin A, and Lindberg D.A.B. Information Rx: evaluation of a new informatics tool for physicians, patients, and libraries. Inf Serv Use. 2006;26(1):1-10. DOI:

https://dx.doi.org/10.3233/isu-2006-26101

2. Prey JE, Woollen J, Wilcox L, Sackeim AD, Hripcsak G, Bakken S, et al. Patient engagement in the inpatient setting: a systematic review. J Am Med Inform Assoc. 2014;21(4):742-50. DOI: https://dx.doi.org/10.1136/amiainl-2013-002141

3. Shipman JP, Kurtz-Rossi S, Funk CJ. The health information literacy research project. J Med Libr Assoc. 2009;97(4):293-301. DOI: http://dx.doi.org/10.3163/1536$\underline{5050.97 .4 .014}$ 
4. McKnight M. Information prescriptions, 1930-2013: an international history and comprehensive review. J Med Libr Assoc. 2014;102(4):271-80. DOI:

https://dx.doi.org/10.3163/1536-5050.102.4.008

5. Calabretta N, Cavanaugh SK. Education for inpatients: working with nurses through the clinical information system. Med Ref Serv Q. 2004;23(2):73-9. DOI:

https://dx.doi.org/10.1300/J115v23n02 07

6. Williams MD, Gish KW, Giuse NB, Sathe NA, Carrell DL. The patient informatics consult service (PICS): an approach for a patient-centered service. Bull Med Libr Assoc. 2001;89(2):185-93.

7. Huber JT, Shapiroll RM, Gillaspy ML. Top down versus bottom up: the social construction of the health literacy movement. The Library Quarterly. 2012;82(4):429-51. DOI: https://dx.doi.org/10.1086/667438

8. Whitney W, Keselman A, Humphreys B. Libraries and librarians: key partners for progress in health literacy research and practice. Stud Health Technol Inform. 2017;240:415-32. DOI: https://dx.doi.org/10.3233/978-1-61499-790-0-415

9. Hansen J. Librarian Consults Through Epic: New Opportunities for Collaboration and Education. Med Ref Serv Q. 2019;38(3):293-9. DOI:

https://dx.doi.org/10.1080/02763869.2019.1623610

10. Witman L. "Information Prescription" orders in the electronic medical record at UVA: bringing our consumer health information service to the bedside and planning for outcomes research. J Hosp Libr. 2017;17(2):137-46. DOI:

https://dx.doi.org/10.1080/15323269.2017.1291049 